\title{
Aberrant methylation of protocadherin 17 and its prognostic value in pediatric acute lymphoblastic leukemia
}

Le Thanh Nha Uyen $\mathrm{MD}^{1}$, Kazuo Sakashita MD, $\mathrm{PhD}^{1,2}$, Lika'a Fasih Y. Al-Kzayer MD, PhD ${ }^{1}$, Yozo Nakazawa MD, $\mathrm{PhD}^{1}$, Takashi Kurata $\mathrm{MD}^{1}$, Kenichi Koike MD, $\mathrm{PhD}^{1}$

${ }^{1}$ Department of Pediatrics, Shinshu University School of Medicine, Matsumoto, Nagano, Japan.

${ }^{2}$ Department of Pediatric Hematology and Oncology, Nagano Children's Hospital, Azumino, Japan.

\section{CORRESPONDING AUTHOR:}

Kazuo Sakashita MD, PhD

Department of Pediatric Hematology and Oncology, Nagano Children’s Hospital

3100 Toyoshina, Azumino city, Nagano, Japan.

Fax: $+81-263-73-5432$

Tel: $+81-263-73-6700$

E-mail: sakasita@shinshu-u.ac.jp

Word count for abstract: 221 words

Word count for main text: 2314 words

Number of tables: 2

Number of figures: 2

Supplemental files: 8

SHORT RUNNING TITLE: PCDH17 methylation in acute lymphoblastic leukemia Keywords: DNA methylation, PCDH17, BCP ALL, relapse, prognostic factors. 

ABBREVIATION
ALL Acute lymphoblastic leukemia
BCP B-cell precursor
BM Bone marrow
COBRA Combined bisulfite restriction analysis
CR Complete remission
DAVID Database for Annotation, Visualization, and Integrated Discovery
EFS Event-free survival
HR Hazard ratio
MNCs Mononuclear cells
OS Overall survival
PB Peripheral blood
RT-PCR Reverse transcriptase PCR
WBC White blood cell 


\begin{abstract}
Background: The outcome of approximately $20 \%$ of acute lymphoblastic leukemia (ALL) patients remains poor because of disease recurrence. We examined whether the DNA methylation of cadherin superfamily genes is a useful biomarker for ALL relapse.

Procedure: We used Infinium Methylation 450K Array to assess the genome-wide DNA methylation status. The methylation status of each individual gene was then determined by combine bisulfite restriction analysis and genome bisulfite sequencing. mRNA expression was evaluated by reverse transcriptase PCR (RT-PCR) and quantitative real-time PCR.

Results: Cadherin superfamily genes including cadherin $(\mathrm{CDH}) 1$, protocadherin $(\mathrm{PCDH}) 8$, and PCDH17 were selected for analyzing the methylation status. In 40 B-cell precursor (BCP) ALL samples at onset, the methylation frequencies of $C D H 1, P C D H 8$, and $P C D H 17$ were $62.5 \%, 55 \%$, and $30 \%$, respectively. $C D H 1$ and $P C D H 8$ methylation was also detected in $80 \%$ and $20 \%$ of control BM samples, respectively. On the contrary, $P C D H 17$ was unmethylated in all control BM samples. There was a significant correlation between the methylation status of $P C D H 17$ (but not $\mathrm{CDH} 1$ and $\mathrm{PCDH} 8$ ) and event-free survival or overall survival. By univariate and multivariate analyses, only $\mathrm{PCDH} 17$ methylation was associated with the increased risk for relapse and mortality in patients with BCP ALL.
\end{abstract}

Conclusion: $\mathrm{PCDH} 17$ methylation at onset was closely related to poor prognosis, and thus it could be used as a new biomarker to predict relapse in BCP ALL. 
The cure rate of childhood acute lymphoblastic leukemia (ALL) has improved over the past four decades. However, the prognosis of approximately $20 \%$ of patients with ALL remains poor

4 because of disease recurrence. A main cause of refractory ALL is the development of 5 chemotherapeutic drug-resistant clone(s).[1-3] The acquisition of chemoresistance is considered

6 to be associated with either genetic or epigenetic alterations.[4-6] Moreover, recent studies

7 demonstrated that relapse clones already present as a small subset of leukemic cells at diagnosis

8 and eventually reemerge as the major clone at relapse.[7] In recent years, many studies have

9 attempted to identify new clinical biomarkers that can predict high-risk patients and serve as 10 targets for novel therapeutic interventions using gene-expression microarrays, DNA-methylation 11 arrays, and next-generation sequencing.[8-11]

12 DNA methylation and histone modifications are the two major epigenetic mechanisms regulating gene expression. Hypermethylation of $\mathrm{CpG}$ islands in the promoter region of tumor suppressor genes, that results in transcriptional silencing, plays an important role in lymphoid-

15 lineage leukemogenesis and may be an important contributor toward relapse.[4, 5] Moreover, DNA methylation profiling is useful for the subtype classification of newly diagnosed ALL patients and prediction of outcome and relapse risk.[12]

Here we present our data demonstrating that the methylation of protocadherin $(P C D H) 17$, a member of non-clustered protocadherins, is related to poor prognosis, and thus proposing PCDH17 methylation as a novel biomarker to predict relapse in B-cell precursor (BCP) ALL.

\section{MATERIALS AND METHODS}

\section{Clinical samples and leukemic cell lines}

This study was approved by the institutional review board of the Shinshu University School of Medicine. Bone marrow (BM) cells were aspirated from 40 children with BCP ALL diagnosed between 1995 and 2008 at Shinshu University Hospital after obtaining informed consent from each of them and/or their parents. The patients were treated according to TCCSG ALL protocol 
(L95-14) between 1995 and 2001, and according to TCCSG ALL protocol (L99-15 and L04-16) between 2002 and 2008. BM mononuclear cells (MNCs) were separated and stored in liquid nitrogen until the time of experiments. In this study, only the samples with $80 \%$ or more leukemic blasts were analyzed. Immunophenotypic diagnosis of BCP ALL was based on either CD10 $\mathrm{CD} 19^{+}$, and cytoplasmic $\mu^{-}$or $\mathrm{CD} 10^{+/-}, \mathrm{CD} 19^{+}$, and cytoplasmic $\mu^{+}$. The following leukemic cell lines were used: Kasumi 2, Kasumi 7, and Kasumi 8 (purchased from the Japanese Collection of Research Bioresources Cell Bank, Ibaraki, Japan); MV4-11, REH, and RCH-ACV (purchased from the German Culture Collection, DSMZ, Braunschweig, Germany). Peripheral blood (PB) MNCs obtained from healthy adult volunteers and BM MNCs obtained from ALL patients in complete remission $(\mathrm{CR})$ were used as controls.

\section{Infinium Methylation 450K Array}

To examine the methylation status of BM samples at diagnosis, $\mathrm{CR}$, and relapse in a relapsed patient with BCP ALL, a global DNA methylation analysis was performed by Takara Bio Inc. (Kusatsu, Japan) using Infinium Methylation 450K Array (Illumina Inc., Tokyo, Japan). The quantitative scores of DNA methylation levels were obtained as beta values determined by Illumina analysis, ranging from 0 for completely unmethylated to 1 for completely methylated. Data were then analyzed using the Database for Annotation, Visualization, and Integrated Discovery (DAVID) bioinformatics software (https://david.ncifcrf.gov) to identify the candidate genes with $\mathrm{CpG}$ sites in which their beta values obtained at relapse were higher than those obtained at diagnosis and CR.

\section{Combined bisulfite restriction analysis (COBRA) and bisulfite sequencing}

Genomic DNA was extracted using the QIAamp DNA Blood Mini Kit (Qiagen, Chuo-ku, Tokyo, Japan), followed by sodium bisulfite treatment using the EZ DNA Methylation Kit (Zymo Research, Irvine, CA, USA) as reported previously.[13, 14] PCR reaction was performed as described previously.[15] 
For combined bisulfite restriction analysis (COBRA), PCR products were digested with Bst UI enzyme (New England BioLabs Japan Inc., Tokyo, Japan), electrophoresed on agarose gels stained with ethidium bromide. The minimal amount of methylated DNA detectable in an unmethylated DNA pool was $20 \%$.

For bisulfite sequencing of the PCDH17 $\mathrm{CpG}$ island, $10 \mu \mathrm{l}$ of each PCR product was electrophoresed onto a 3\% agarose gel. The bands were then excised and purified using the Geneclean 2 Kit (Bio 101 Inc., La Jolla, CA, USA). Purified PCR products were cloned into the pGEM-T Easy vector (Promega Corp., Madison, WI, USA). The plasmids were extracted using the Wizard Plus Minipreps DNA Purification System (Promega). Individual clones were sequenced on an ABI PRISM 3100 Genetic Analyzer (Applied Biosystems) using a DYEnamic ET Terminator Cycle Sequencing Kit (Amersham Biosciences, Piscataway, NJ, USA).

\section{Reverse transcriptase PCR (RT-PCR) and quantitative real-time PCR}

Total RNA was isolated using the RNeasy Mini Kit (Qiagen), and cDNA was prepared using PrimeScript II first strand cDNA Synthesis Kit (Takara Bio Inc.). The cDNA was used as a template for RT-PCR, and quantitative real-time PCR with primers and probes listed in Supplemental Table S2. RT-PCR was performed as reported previously.[15] Real-time PCR was carried out in an ABI sequence detection system using the Master Mix (Applied Biosystems). Each assay was performed in triplicates for each sample.

\section{Multiplex ligation-dependent probe amplification (MLPA)}

IKZF1 deletion was identified by MLPA. The MLPA assays, using SALSA MLPA P202 IKZF1 kit (MRC-Holland, Amsterdam, The Netherlands), were performed according to the manufacturer's protocol. The data were analyzed by Coffalyser.Net software (free download at https://www.mlpa.com, MRC-Holland).

\section{Statistical analyses}

The chi-square or Fisher's exact tests was used to determine the association of the methylation of $\mathrm{PCDH} 17$ with that of other cadherin superfamily genes, and to compare their methylation 


8

status at diagnosis with that at relapse. Spearman's rank correlation test was performed to determine the relationship between two variables.

Actuarial estimates of event-free survival (EFS) and overall survival (OS) were calculated using the Kaplan-Meier method. Patients were censored for EFS if they were alive and in remission at last follow-up, and were censored for OS if they were alive at last follow-up. Follow-up data were available through December 31, 2014. Risk factors for relapse or mortality were applied to the univariate and multivariate Cox proportional hazards regression analysis. All $P$ values were two-sided and $P$ values 0.05 or less were considered statistically significant. All statistical analyses were performed with EZR (Saitama Medical Center, Jichi Medical University; http://www.jichi.ac.jp/saitama-sct/SaitamaHP.files/statmedEN.html;Kanda, 2012).

\section{RESULTS}

\section{The methylation status of cadherin superfamily genes in BCP ALL}

The genome-wide DNA methylation status of BM samples (at diagnosis, CR, and relapse) of a 2-year-old male diagnosed with BCP ALL was examined, followed by functional analysis of large gene lists using bioinformatics DAVID program which identified cadherin superfamily genes as the top candidate genes with aberrant methylation at relapse (Supplemental Appendix S1). Among these cadherin superfamily genes, Cadherin (CDH)1, $P C D H 8$, and $P C D H 17$ were previously demonstrated as tumor suppressor genes and frequently detected as hypermethylated in various cancers such as gastric, esophageal squamous cell, hepatocellular, and breast cancers. [16-19] Therefore, we selected these 3 genes for further analysis. First, we examined the methylation status of $C D H 1, P C D H 8$, and $P C D H 17$ in 6 ALL cell lines, in 40 newly diagnosed Ph1-negative BCP ALL samples obtained at diagnosis, and in control PB and BM cells using COBRA method. The results are shown in Fig 1. CDH1 and PCDH8 methylation was observed in all cell lines, whereas $P C D H 17$ methylation was detected in 4 of them. $C D H 1, P C D H 8$, and PCDH17 were methylated in $62.5 \%, 55 \%$, and $30 \%$ of ALL cases, respectively. However, 
methylation of $\mathrm{PCDH} 8$ and $\mathrm{CDH1}$ was also detected in $80 \%$ and $20 \%$ of control $\mathrm{BM}$ samples, respectively. These findings were partly due to the methylation of CD71-positive BM cells. In contrast, $\mathrm{PCDH} 17$ was unmethylated in control BM cells. There was a significant association between $C D H 1$ methylation and $P C D H 8$ methylation $(P<0.001)$. Furthermore, $P C D H 17$ methylation was associated with both $C D H 1$ and $P C D H 8$ methylation $(P=0.018$ and $P=0.013$, respectively).

Thereafter, we compared the methylation status of $31 \mathrm{PCDH} 17 \mathrm{CpG}$ sites at diagnosis and at relapse in 3 patients using bisulfite sequencing. As presented in Supplemental Figure S2, total methylated $\mathrm{PCDH} 17$ sites obtained from 8 clones increased significantly at relapse, as compared with those obtained from clones at diagnosis in 2 of 3 patients $(P<0.0001)$.

We examined the relationship between the methylation status and the mRNA expression of PCDH17 in various cell types. $P C D H 17$ methylation was almost negligible in control PB cells, whereas a total number of methylated sites varied among ALL samples and ALL cell lines, as determined by bisulfite sequencing (Supplemental Figure S3). Control PB cells expressed PCDH17 mRNA due to the present of PB CD33+ cells, whereas the mRNA was variable in ALL samples and cell lines (Supplemental Figure S4). There was no correlation between PCDH17 methylation and mRNA expression levels (Supplemental Figure S5).

The relationship between methylation status of cadherin superfamily genes at diagnosis and clinical characteristics of patients with BCP ALL

We then determined whether the methylation status of $C D H 1, P C D H 8$, and $P C D H 17$ at diagnosis examined by COBRA was associated with the clinical characteristics of BCP ALL. As shown in Table 1, PCDH17 methylation was significantly correlated with high white blood cell (WBC) counts $(P=0.047)$, however, no correlation was found with age, gender, genetic abnormalities including IKZF1 deletion, $\mathrm{t}(4 ; 11)$ and $\mathrm{t}(12 ; 21)$, or steroid response. There were substantial relationships between $C D H 1$ methylation and male sex, as well as between $P C D H 8$ methylation and both male sex and high WBC. The EFS and OS of 14 patients treated between 
1995 and 2001 were similar to those of 26 patients treated between 2002 and 2008 [10-year EFS, 64.3\% (95\% CI: $34.3-83.3)$ vs. $61.5 \%$ (95\% CI: 40.3-77.1)]; 10-year OS, 70.7\% (95\% CI: $39.4-$ 87.9) vs. $72.5 \%$ (95\% CI: 59.5-91.4), although ALL protocols were different between the two groups. Thus, we analyzed the impact of the methylation of cadherin superfamily genes on the outcomes of all 40 patients. EFS of $\mathrm{PCDH17}$ methylation-positive group was profoundly inferior to that of $P C D H 17$ methylation-negative group: $33 \%$ (95\% CI, 10-59) vs. $75 \%$ (95\% CI, 55-87); $P=0.005$ (Fig 2). A significant difference in OS was also found between the two groups: $50 \%$ (95\% CI, 21-73) vs. $82 \%(95 \% \mathrm{CI}, 62-92) ; P=0.016$. Conversely, there were no substantial correlations between the methylation status of $C D H 1$ and EFS or OS, and between the methylation status of $\mathrm{PCDH8}$ and EFS or OS.

As shown in Table 2, univariate analysis revealed that $\mathrm{PCDH17}$ methylation was associated with increased risk for relapse and mortality in patients with BCP ALL [hazards ratio (HR), 3.85; $P=0.001$ and HR, 5.05; $P=0.017$, respectively]. According to multivariate analysis, only PCDH17 methylation was related to poor outcome [HR, 5.23; $P=0.016$ for relapse and HR, 8.22; $P=0.016$ for mortality]. However, $C D H 1$ and $P C D H 8$ methylation did not influence ALL prognosis.

\section{DISSCUSSION}

DNA hypermethylation plays a significant role in the leukemogenesis and hematologic relapse in ALL. [4, 5] For a comprehensive evaluation of the DNA methylation in a pediatric patient with ALL, we performed a genome-wide DNA methylation analysis. The patient already harbored the relapse clone as a minor population at diagnosis. Therefore, to identify the candidate genes associated with relapse, we selected genes that were methylated at higher levels at relapse than at onset. As a result, cadherin superfamily genes, including $C D H 1, P C D H 8$, and $P C D H 17$, were the top gene-family in this patient. Among 40 ALL samples at onset, the methylation frequencies of CDH1, $\mathrm{PCDH} 8$, and $\mathrm{PCDH} 17$ were $62.5 \%, 55 \%$, and $30 \%$, respectively. The methylation of CDH1 and $P C D H 8$ was also detected in $80 \%$ and $20 \%$ of control BM samples, respectively. On 
the contrary, $P C D H 17$ was unmethylated in all 10 control BM samples. These results suggest that PCDH17 methylation is a specific phenomenon for ALL blasts at onset.

In our study, methylation of $P C D H 8$ and $C D H 1$ in BM cells was detected in CD33+/CD71+ cells. CD71 was expressed on proliferating cells. Myeloid BM precursors from dividing blast cells to myelocytes were variably CD71 positive, whereas non-dividing late precursors were CD71 negative.[20-22] Therefore, the methylation of $P C D H 8$ and $C D H 1$ in CD33+/CD71+ cells may be related to the proliferative status of myeloid precursors.

Interestingly, the Kaplan-Meier estimates showed that EFS and OS of the PCDH17 methylation-positive group were significantly lower than those of the $P C D H 17$ methylationnegative group. Conversely, there were no significant correlations between the methylation status of either $\mathrm{CDH} 1$ or $\mathrm{PCDH} 8$ and EFS or OS. According to univariate and multivariate analysis, PCDH17 methylation, but not $C D H 1$ or $P C D H 8$ methylation, was associated with an increased risk for relapse or mortality in BCP ALL patients. These results suggest that PCDH17 methylation at onset is related to poor prognosis and thus it could be considered as a new biomarker to predict relapse in such subset of patient. However, a larger study is necessary to clarify this issue. $\mathrm{PCDH} 17$ is a member of non-clustered protocadherin, a subfamily of cadherin superfamily genes.[23] $P C D H 17$ is frequently downregulated through the promoter hypermethylation in a number of cancers.[24-26] The restoration of $P C D H 17$ expression reduces cell proliferation and migration in esophageal squamous cell carcinoma, [17] and induces tumor cell apoptosis and autophagy in gastric and colorectal cancers.[18] However, there was no correlation between the methylation status and the mRNA expression level of PCDH17 in patients with BCP ALL in this study, implying the involvement of alternative mechanisms in regulation of $\mathrm{PCDH} 17$ gene expression. Accordingly, the association of $\mathrm{PCDH} 17$ methylation with poor prognosis may occur through a mechanism that are independent of methylationmediated transcriptional silencing. 
In our genome-wide DNA methylation analysis of one relapsed case with BCP ALL, the 182 relapse-mediated increases in beta values of $P C D H 10, C D H 11$, and $C D H 13$ methylation were 183 less than 0.3 . Therefore, we excluded these 3 genes from the subsequent candidate gene analyses 184 in this study. However, Narayan et al. identified $P C D H 10$ hypermethylation in approximately $18580 \%$ of patients with B-ALL.[27] Hogan et al. found that $C D H 1, C D H 11$, and $C D H 13$ were 186 hypermethylated and downregulated in relapsed pediatric ALL cases.[5] Considering one single 187 sample with a clonal expansion of a minor clone at relapse, we cannot completely rule out the 188 impact of methylation of cadherin superfamily genes other than $P C D H 17$ and the other family 189 genes on ALL relapse.

\section{Acknowledgements}

This work was supported by Grants-in-Aid for Scientific Research (No. 26461575 and No. 15H04874) from the Ministry of Education of Japan and Friends of Leukemia Research Fund.

\section{Author contributions}

L.T.N.U performed the research, analyzed the data and wrote the paper. K.S. contributed to data management and statistical analysis and wrote the paper. K.K. revised the manuscript and contributed to paper writing. Y.N., T.K., and A.L.F.Y assisted in doing experiments.

\section{Conflict of interest}

The authors declare no potential conflicts of interest

\section{REFERENCES}

1. Pui C-H, Relling MV, Downing JR. Acute lymphoblastic leukemia. New England Journal of Medicine 2004;350:1535-48.

2. Ross D. Novel mechanisms of drug resistance in leukemia. Leukemia 2000;14:467-73. 
3. Swerts K, De Moerloose B, Dhooge C, Laureys G, Benoit Y, Philippé J. Prognostic significance of multidrug resistance-related proteins in childhood acute lymphoblastic leukaemia. European Journal of Cancer 2006;42:295-309.

4. Bhatla T, Wang J, Morrison DJ, Raetz EA, Burke MJ, Brown P, Carroll WL. Epigenetic reprogramming reverses the relapse-specific gene expression signature and restores chemosensitivity in childhood B-lymphoblastic leukemia. Blood 2012;119:5201-10.

5. Hogan LE, Meyer JA, Yang J, Wang J, Wong N, Yang W, Condos G, Hunger SP, Raetz E, Saffery R. Integrated genomic analysis of relapsed childhood acute lymphoblastic leukemia reveals therapeutic strategies. Blood 2011;118:5218-26.

6. Pui C-H, Mullighan CG, Evans WE, Relling MV. Pediatric acute lymphoblastic leukemia: where are we going and how do we get there? Blood 2012;120:1165-74.

7. Henderson MJ, Choi S, Beesley AH, Sutton R, Venn NC, Marshall GM, Kee UR, Haber M, Norris MD. Mechanism of relapse in pediatric acute lymphoblastic leukemia. Cell Cycle 2008;7:1315-20.

8. Figueroa ME, Chen S-C, Andersson AK, Phillips LA, Li Y, Sotzen J, Kundu M, Downing JR, Melnick A, Mullighan CG. Integrated genetic and epigenetic analysis of childhood acute lymphoblastic leukemia. The Journal of clinical investigation 2013;123:3099-11.

9. Leshchenko VV, Kuo P-Y, Shaknovich R, Yang DT, Gellen T, Petrich A, Yu Y, Remache Y, Weniger MA, Rafiq S. Genomewide DNA methylation analysis reveals novel targets for drug development in mantle cell lymphoma. Blood 2010;116:1025-34.

10. Roberts KG, Li Y, Payne-Turner D, Harvey RC, Yang Y-L, Pei D, McCastlain K, Ding L, Lu C, Song G. Targetable kinase-activating lesions in Ph-like acute lymphoblastic leukemia. New England Journal of Medicine 2014;371:1005-15.

11.Tayrac Md, Etcheverry A, Aubry M, Saïkali S, Hamlat A, Quillien V, Treut AL, Galibert MD, Mosser J. Integrative genome-wide analysis reveals a robust genomic glioblastoma signature 
associated with copy number driving changes in gene expression. Genes, Chromosomes and Cancer 2009;48:55-68.

12. Milani L, Lundmark A, Kiialainen A, Nordlund J, Flaegstad T, Forestier E, Heyman M, Jonmundsson G, Kanerva J, Schmiegelow K. DNA methylation for subtype classification and prediction of treatment outcome in patients with childhood acute lymphoblastic leukemia. Blood 2010;115:1214-25.

13. Sakashita K, Koike K, Kinoshita T, Shiohara M, Kamijo T, Taniguchi Si, Kubota T. Dynamic DNA methylation change in the $\mathrm{CpG}$ island region of $\mathrm{p} 15$ during human myeloid development. Journal of Clinical Investigation 2001;108:1195-204.

14. Zhao XY, Sakashita K, Kamijo T, Hidaka E, Sugane K, Kubota T, Koike K. Granulocytemacrophage colony-stimulating factor induces de novo methylation of the p15 CpG island in hematopoietic cells. Cytokine 2005;31:203-12.

15. Al-Kzayer LaFY, Sakashita K, Matsuda K, Al-Hadad SA, Al-Jadiry MF, Abed WM, Abdulkadhim JM, Al-Shujairi TA, Hasan JG, Al-Abdullah HMS. Genetic evaluation of childhood acute lymphoblastic leukemia in Iraq using FTA cards. Pediatric blood \& cancer 2012;59:461-7.

16. Miyamoto K, Fukutomi T, Akashi-Tanaka S, Hasegawa T, Asahara T, Sugimura T, Ushijima T. Identification of 20 genes aberrantly methylated in human breast cancers. International journal of cancer 2005; 116:407-14.

17. Haruki S, Imoto I, Kozaki K-i, Matsui T, Kawachi H, Komatsu S, Muramatsu T, Shimada Y, Kawano T, Inazawa J. Frequent silencing of protocadherin 17, a candidate tumour suppressor for esophageal squamous-cell carcinoma. Carcinogenesis 2010:1027-36.

18. Hu X, Sui X, Li L, Huang X, Rong R, Su X, Shi Q, Mo L, Shu X, Kuang Y. Protocadherin 17 acts as a tumour suppressor inducing tumour cell apoptosis and autophagy, and is frequently methylated in gastric and colorectal cancers. The Journal of pathology 2013;229:62-73. 
19. Jiang L, Chan JY-W, Fung K-P. Epigenetic loss of CDH1 correlates with multidrug resistance in human hepatocellular carcinoma cells. Biochemical and biophysical research communications 2012; 422: 739-44.

20. Blair A, Hogge D, Sutherland H. Most acute myeloid leukemia progenitor cells with longterm proliferative ability in vitro and in vivo have the phenotype CD34+/CD71-/HLA-DR-. Blood 1998;92:4325-35.

21. Sieff C, Bicknell D, Caine G, Robinson J, Lam G, Greaves M. Changes in cell surface antigen expression during hemopoietic differentiation. Blood 1982;60:703-13.

22. Liu Q, Wang M, Hu Y, Xing H, Chen X, Zhang Y, Zhu P. Significance of CD71 expression by flow cytometry in diagnosis of acute leukemia. Leukemia \& lymphoma 2014;55:892-8.

23. Kim S-Y, Yasuda S, Tanaka H, Yamagata K, Kim H. Non-clustered protocadherin. Cell adhesion \& migration 2011;5:97-105.

24. Costa VL, Henrique R, Danielsen SA, Eknaes M, Patrício P, Morais A, Oliveira J, Lothe RA, Teixeira MR, Lind GE. TCF21 and PCDH17 methylation: An innovative panel of biomarkers for a simultaneous detection of urological cancers. Epigenetics 2011;6:1120-30.

25. Lin Y-L, Xie P-G, Wang L, Ma J-G. Aberrant methylation of protocadherin 17 and its clinical significance in patients with prostate cancer after radical prostatectomy. Med Sci Monit 2014;20:1376-82.

26. Sui X, Wang D, Geng S, Zhou G, He C, Hu X. Methylated promoters of genes encoding protocadherins as a new cancer biomarker family. Molecular biology reports 2012;39:110511.

27. Narayan G, Freddy AJ, Xie D, Liyanage H, Clark L, Kisselev S, Un Kang J, Nandula SV, McGuinn C, Subramaniyam S. Promoter methylation-mediated inactivation of PCDH10 in acute lymphoblastic leukemia contributes to chemotherapy resistance. Genes, Chromosomes and Cancer 2011;50:1043-53. 


\section{Figure legends}

Figure 1. Methylation status of $\mathrm{CDH}, \mathrm{PCDH8}$, and $\mathrm{PCDH17}$ by combined bisulfite restriction analysis (COBRA)

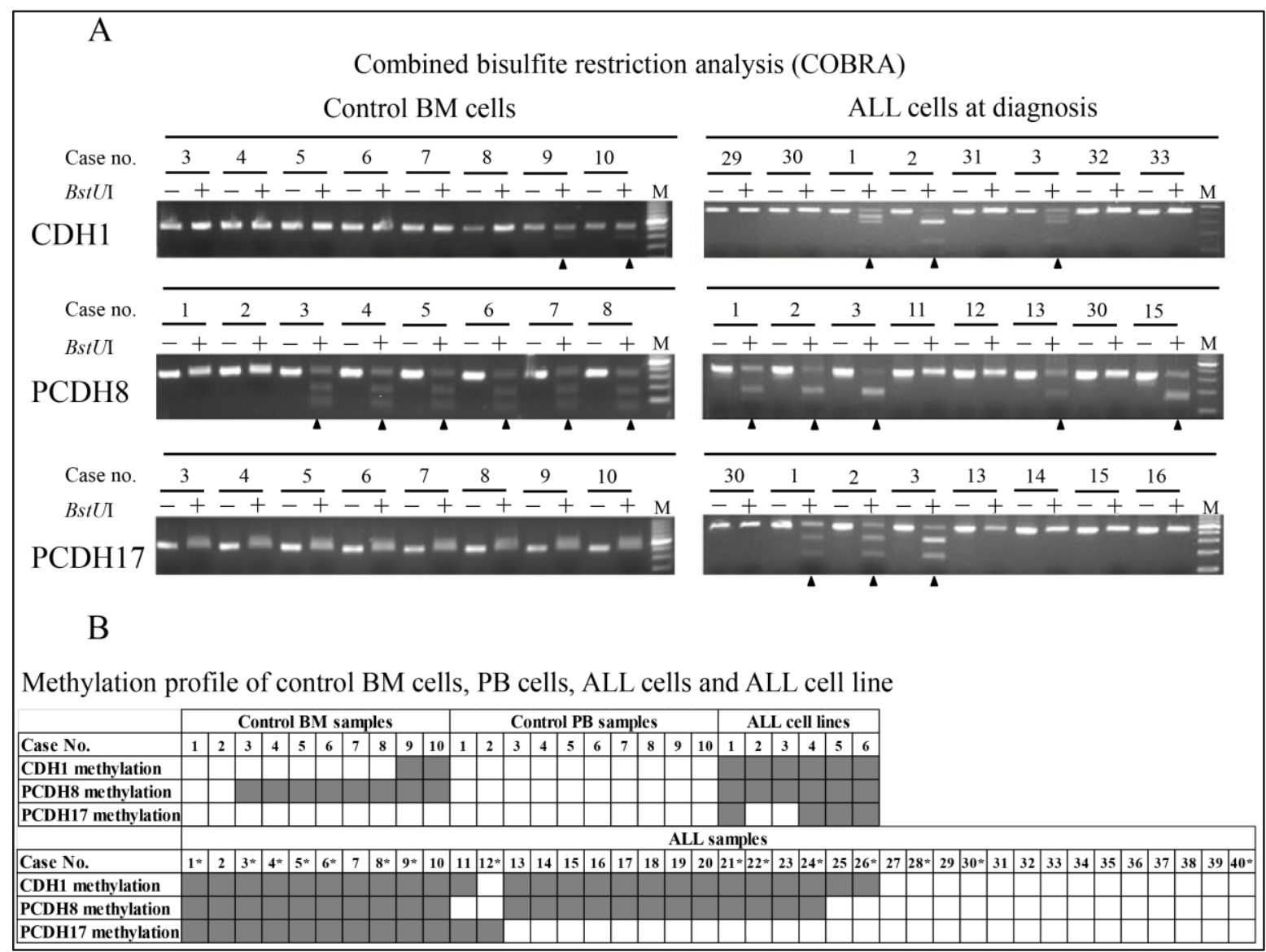

(A) Representative results of COBRA analysis: PCR products of promoter $C D H 1, P C D H 8$, and PCDH17 of control BM cells and ALL samples were digested with BstUI enzyme, subjected to $2 \%$ agarose gel stained with ethidium bromide. The black triangle symbols indicate methylated samples with "CGCG" BstUI enzyme cut site (digested in smaller fragments). The "minus" symbols represent no BstUI treatment, and the "plus" symbols represent Bst UI treatment. The letter "M" represents DNA size marker. (B) The summary of the methylation profile of control BM, PB samples, ALL cell lines and 40 newly diagnosed ALL samples. Gray squares: cases with methylated genes. ${ }^{*}$ : relapsed case. 
Figure 2. Kaplan-Meier survival analysis of ALL children with or without methylation of the three cadherin superfamily genes

B

Kaplan-Meier estimates of EFS and OS were compared between methylated and unmethylated CDH1 (A), PCDH8 (B), and PCDH17 (C) determined by COBRA. 


\section{Supplemental Figure legends}

Supplemental Figure S1. Comparison of $\mathrm{IgH}$ rearrangements at diagnosis and relapse in a BCP ALL patient

\section{Supplemental Figure S1}

A

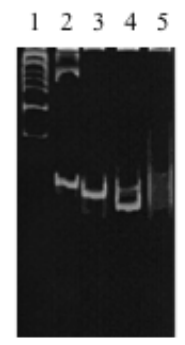

B

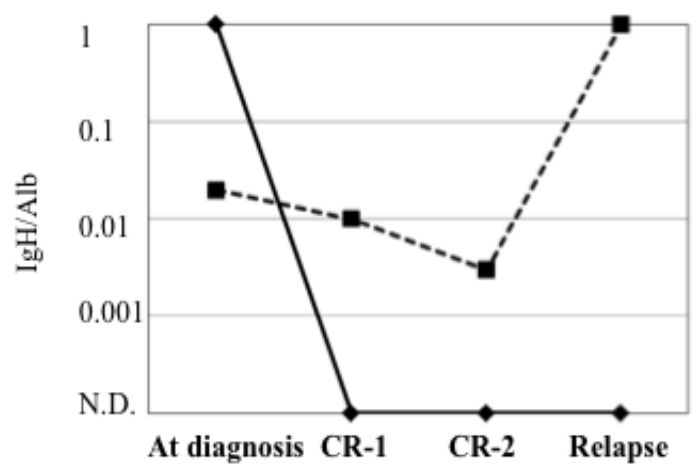

Comparison of $\mathrm{IgH}$ rearrangements at diagnosis and relapse in a BCP ALL patient

(A) Electrophoresis of $I g H$ (CDR-III region) gene rearrangements. Lane 1: DNA size marker, lane 2: Raji cells, lane 3: one band indicating the presence of a clonal $\mathrm{IgH}$ gene rearrangement at diagnosis, lane 4: two bands indicating the presence of two clonal rearrangements at relapse, and lane 5: negative control (normal PB). (B) Real-time PCR-based detection of two leukemic clones with different $\lg H$ gene rearrangements at diagnosis, $\mathrm{CR}$, and relapse. Albumin $(A L B)$ gene was used for normalization, and the values were expressed as relative quantities compared with the value at diagnosis or relapse.

(A) Electrophoresis of $\operatorname{IgH}$ (CDR-III region) gene rearrangements. (B) Real-time PCR-based detection of two leukemic clones with different $\operatorname{IgH}$ gene rearrangements at diagnosis, $\mathrm{CR}$, and relapse. 
Supplemental Figure S2. Allelic methylation status of the PCDH17 CpG islands by bisulfite sequencing

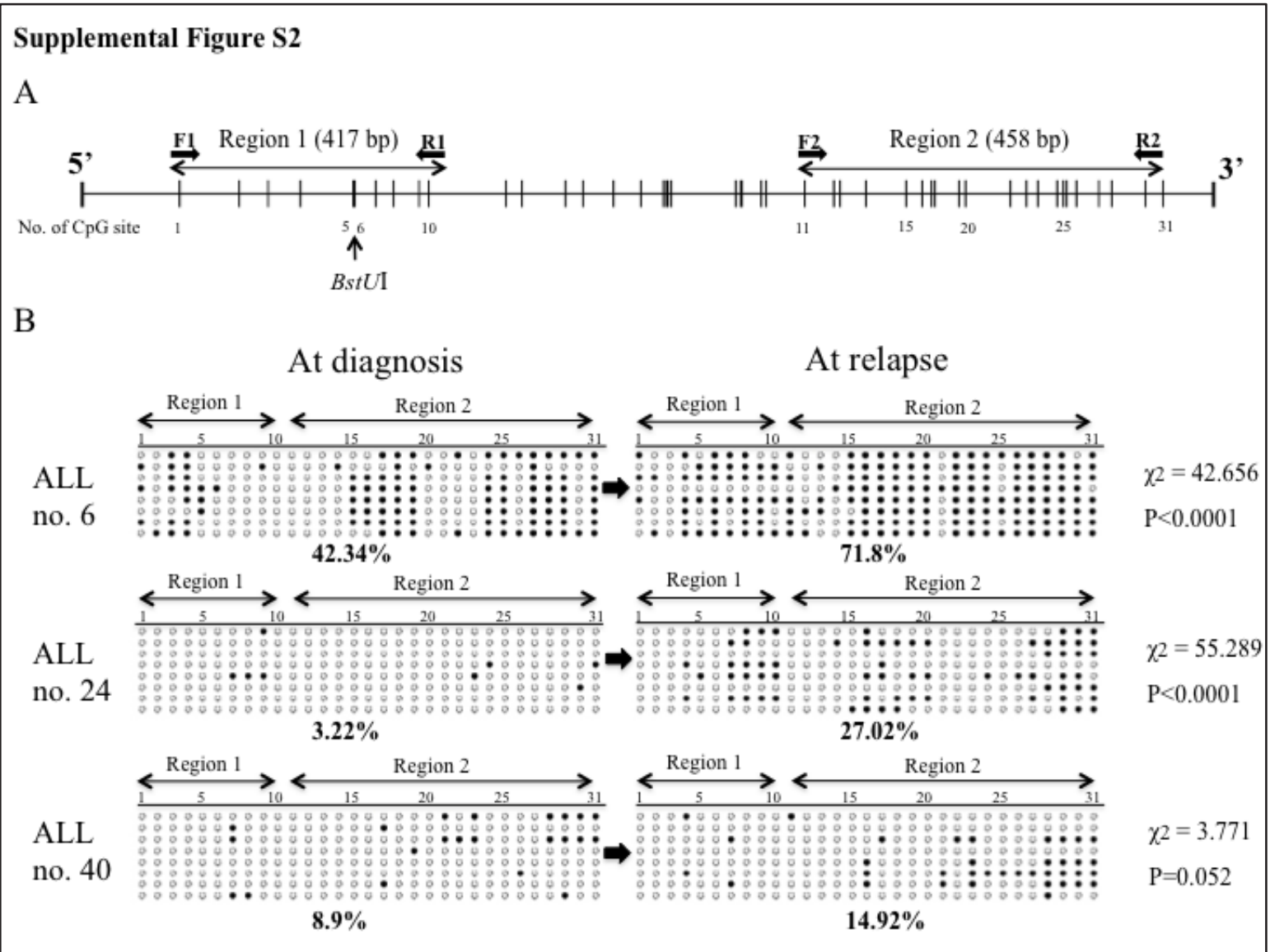

(A) A schematic map of the $\mathrm{CpG}$ island region around exon 1 of $P C D H 17$. Vertical black bars indicate the location of individual $\mathrm{CpG}$ sites. The horizontal arrows indicate the position of two examined fragments (region 1 and region 2). The vertical arrow indicates a restriction site for the Bst UI enzyme. The binding sites of the primers used in this experiment are also shown (F1 and $\mathrm{R} 1, \mathrm{~F} 2$ and R2). (B) The allelic methylation status of the $P C D H 17 \mathrm{CpG}$ island at diagnosis and at relapse among 3 ALL cases. Each horizontal row represents an individual cloned and sequenced allele following bisulfite treatment. Methylated $\mathrm{CpG}$ sites are marked as closed circles and unmethylated sites as open circles. Numbers above horizontal rows correspond to those of the CpG sites of $P C D H 17$ shown in (A). 
Supplemental Figure S3.

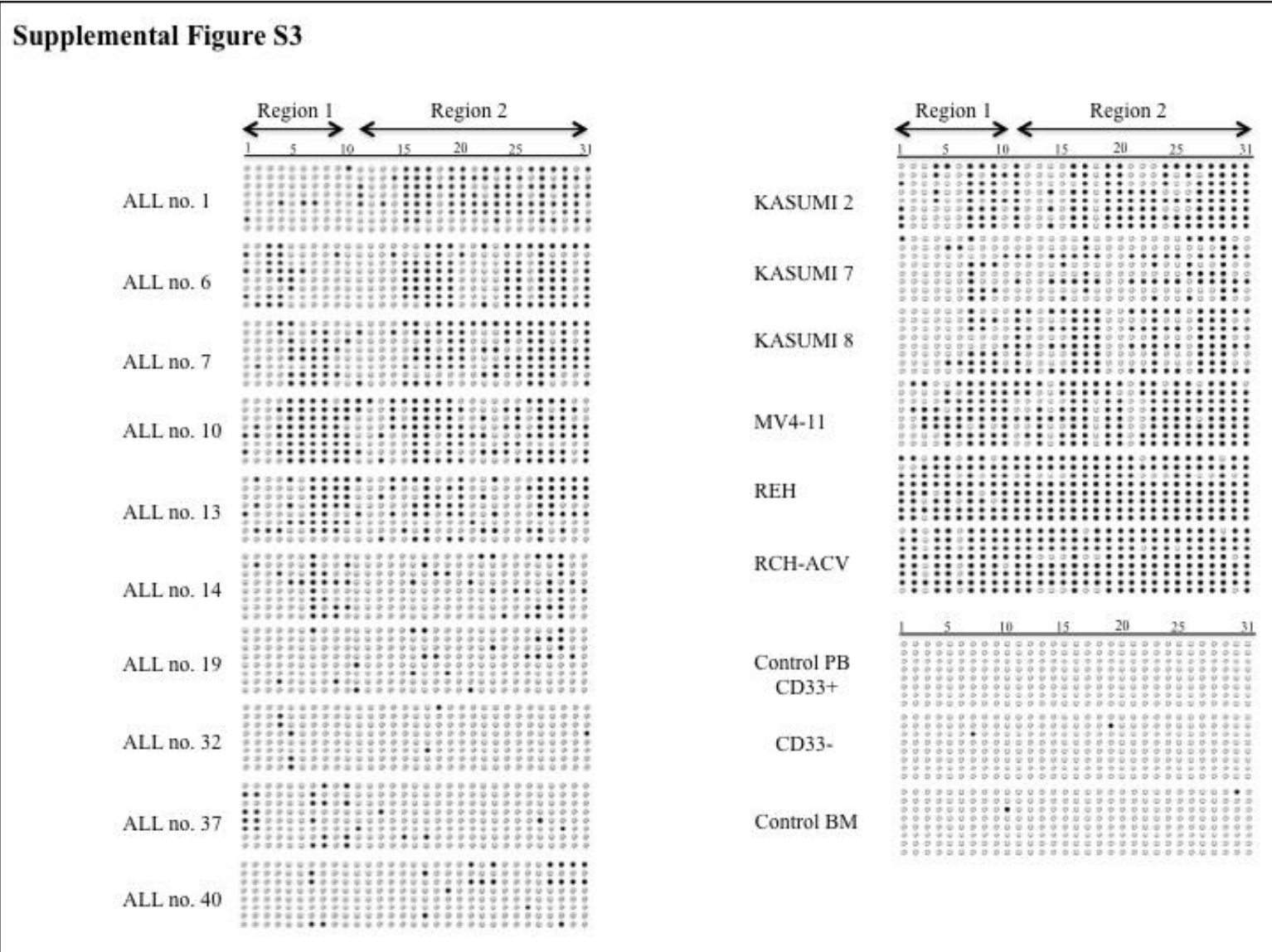

Allelic methylation status of $P C D H 17$ individual $\mathrm{CpG}$ site by bisulfite sequencing in ALL cases, ALL cell lines and normal blood cells. 


\section{Supplemental Figure S4.}

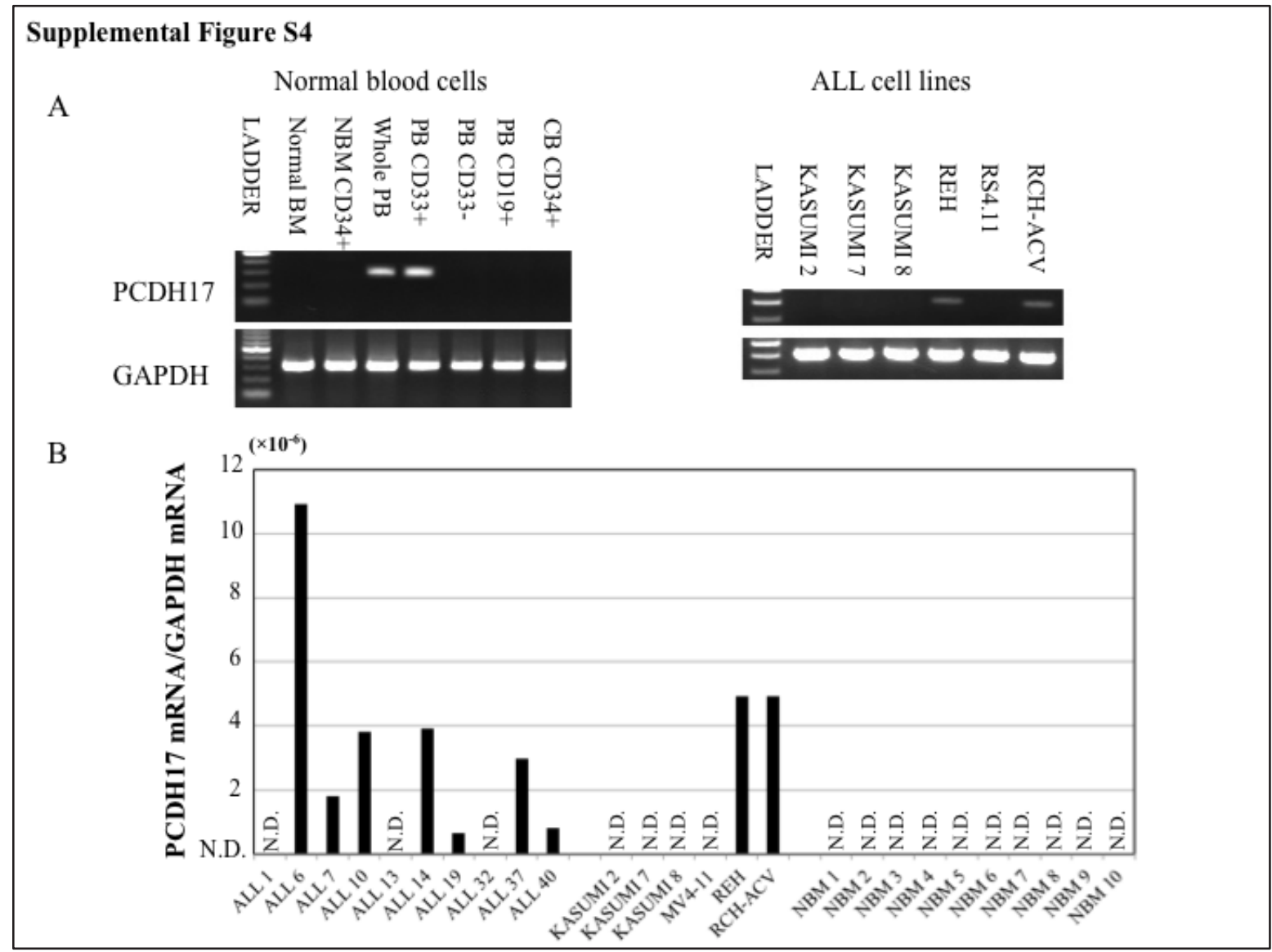

PCDH17 mRNA expression examined by RT-PCR (A) and realtime PCR (B) in normal blood cells, ALL cell lines and ALL samples. The expression levels are displayed as ratios of PCDH17 and GAPDH. N.D., not detectable. 


\section{Supplemental Figure S5.}

\section{Supplemental Figure S5}

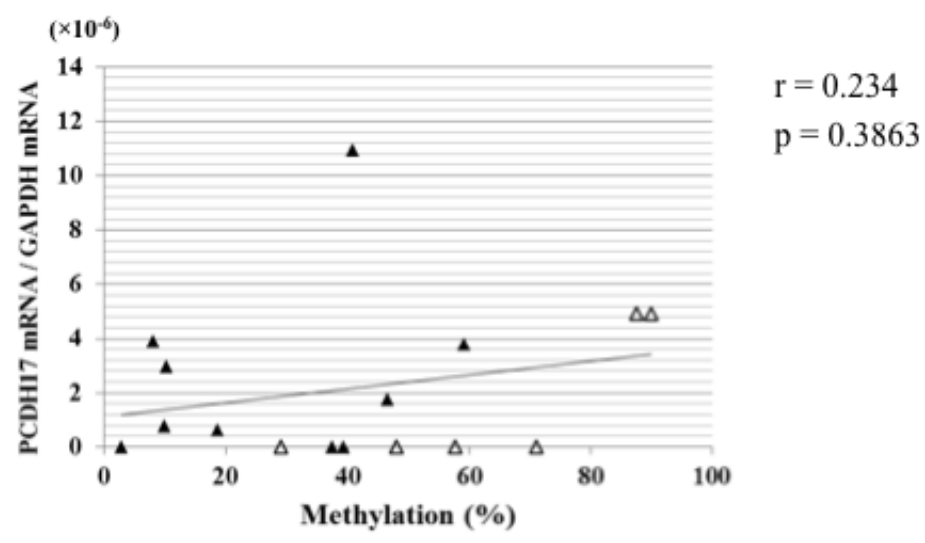

No correlation between methylation status and mRNA expression of PCDH17 among 10 ALL cases and 6 ALL cell lines. Closed triangles, ALL patient samples; open triangles, ALL cell line samples.

Relationship between $P C D H 17$ methylation and expression levels. Closed triangles: ALL patient samples; open triangles: ALL cell line samples 


\section{Tables}

TABLE 1. Relationship between methylation status of cadherin superfamily genes at diagnosis and clinical characteristics of patients with BCP ALL

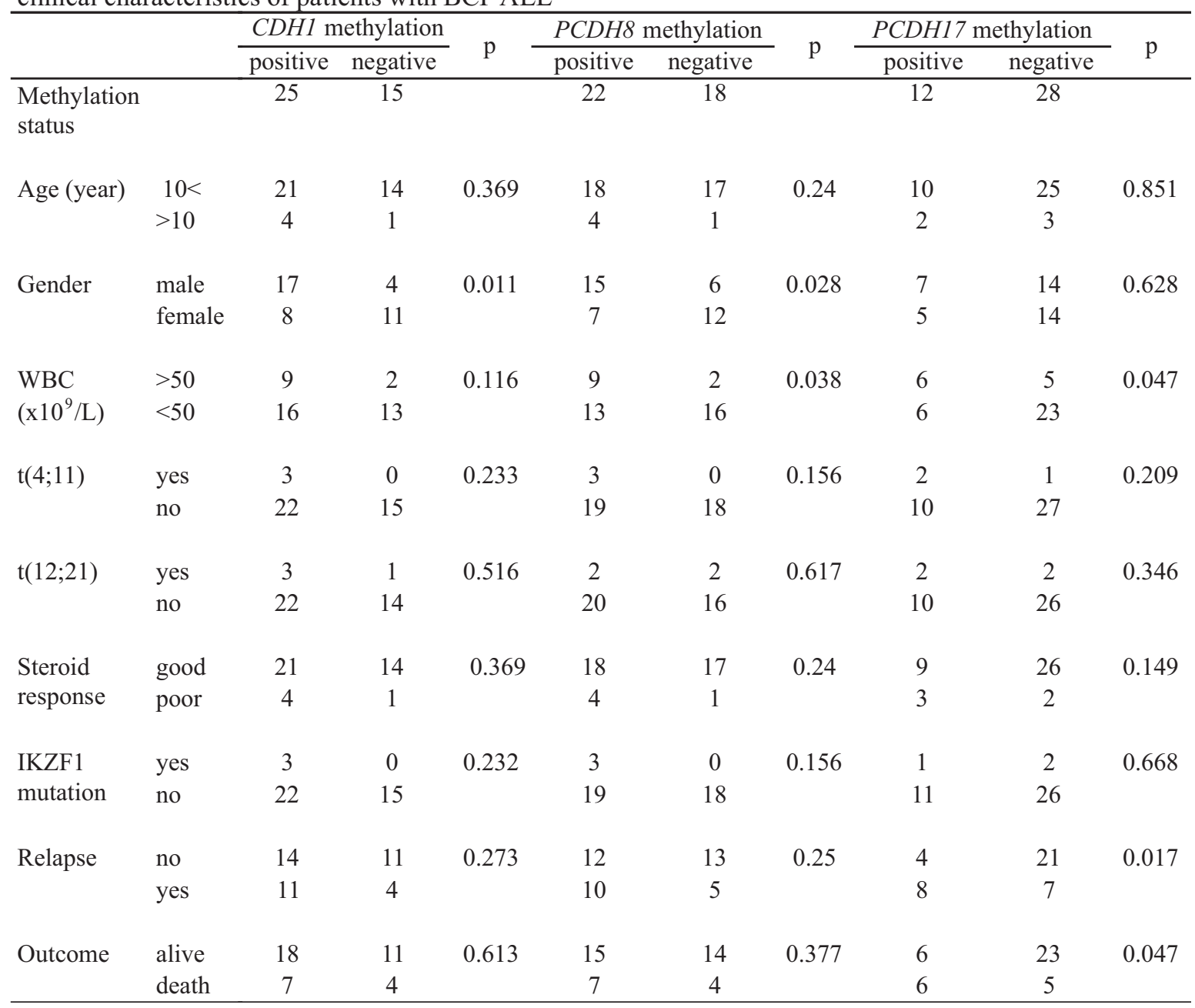

TABLE 2. Univariate and multivariate analyses of risk factors for outcomes

\begin{tabular}{|c|c|c|c|c|c|c|c|c|}
\hline & \multicolumn{4}{|c|}{ Relapse } & \multicolumn{4}{|c|}{ Overall survival } \\
\hline & \multicolumn{2}{|c|}{ Univariate analysis } & \multicolumn{2}{|c|}{ Multivariate analysis } & \multicolumn{2}{|c|}{ Univariate analysis } & \multicolumn{2}{|c|}{ Multivariate analysis } \\
\hline & $\mathrm{HR}(95 \% \mathrm{CI})$ & $\mathrm{p}$ & $\mathrm{HR}(95 \% \mathrm{CI})$ & $\mathrm{p}$ & $\operatorname{HR}(95 \% \mathrm{CI})$ & $\mathrm{p}$ & $\mathrm{HR}(95 \% \mathrm{CI})$ & $\mathrm{p}$ \\
\hline $\mathrm{WBC}\left(<50 \times 10^{9} / \mathrm{L} / \geq 50 \times 10^{9} / \mathrm{L}\right)$ & $1.09(0.346-3.43)$ & 0.885 & - & $>0.1$ & $1.75(0.511-5.974)$ & 0.373 & - & $>0.1$ \\
\hline Age $(<10$ years $/ \geq 10$ years $)$ & $1.04(0.917-1.17)$ & 0.57 & - & $>0.1$ & $2.01(0.434-9.329)$ & 0.372 & - & $>0.1$ \\
\hline Steroid response (good / poor) & $1.36(0.306-6.016)$ & 0.69 & - & $>0.1$ & $1.69(0.364-7.81)$ & 0.505 & - & $>0.1$ \\
\hline $\mathrm{t}(4 ; 11)$ (negative / positive) & $1.04(0.136-7.902)$ & 0.971 & - & $>0.1$ & $1.57(0.20-12.27)$ & 0.669 & - & $>0.1$ \\
\hline $\mathrm{t}(12 ; 21)$ (negative / positive) & $0.51(0.067-3.908$ & 0.52 & - & $>0.1$ & -* & - & $-*$ & - \\
\hline IKZF1 mutation (negative /posotive) & $2.14(0.482-9.511)$ & 0.317 & - & $>0.1$ & $1.03(0.132-8.082)$ & 0.975 & - & $>0.1$ \\
\hline PCDH17 methylation (negative / positive) & $3.85(1.39-10.64)$ & 0.001 & $5.23(1.355-20.19)$ & 0.016 & $5.05(1.334-19.090)$ & 0.017 & $8.22(1.484-45.53)$ & 0.016 \\
\hline PCDH8 methylation (negative / positive) & $1.74(0.593-5.085)$ & 0.313 & - & $>0.1$ & $1.53(0.447-5.213)$ & 0.5 & - & $>0.1$ \\
\hline CDH1 methylation (negative / positive) & $1.75(0.558-5.507)$ & 0.337 & - & $>0.1$ & $1.07(0.314-3.67)$ & 0.901 & - & $>0.1$ \\
\hline
\end{tabular}

